\title{
ON THE K-LINE WIDTH-ABSOLUTE MAGNITUDE RELATION
}

\author{
M. K. V. BAPPU \\ Indian Institute of Astrophysics, Kodaikanal, India
}

\begin{abstract}
Bright fine mottles seen on the solar disk are identified as the agency that locates the Sun on the K-line width-absolute magnitude relation of Wilson and Bappu. The contribution of the supergranular network emission and the plage emission from centres of activity tends to upset the uniqueness of the relation. The line width-absolute magnitude relation is thus a characteristic of the convection zone underlying the stellar atmosphere.
\end{abstract}

Perhaps the most striking characteristic of the K-line emission from stellar chromospheres is the relationship between the width of the line and the visual absolute magnitude of the star that is valid over a range of about fifteen magnitudes. Indeed, in itself it is very suggestive of a common astrophysical characteristic operative in these atmospheres over the entire range that experiences an increase in magnitude of its impulsive scope as one goes from stars of fainter visual luminosity to the brighter ones. The Sun fits this relation well and since it is the only star which presents a disk for detailed scrutiny, much interest centres on an identification on the solar disc of that feature which gives rise to the $\mathrm{K}$-line width-absolute magnitude relation.

A calcium spectroheliogram of the solar disc shows primarily three principal characteristics. If we have a centre of activity on the visible portion at the time, it will manifest itself with enhanced calcium emission over a sizeable region and this we term a calcium plage. Then, there is the network of calcium emission which has been shown by Leighton to coincide with the boundaries of the supergranulation. And within the configuration of a network cell, we come across localized bright points of emission which we recognize as bright fine mottles. The boundary of the supergranule has enhanced emission with a twofold contribution. The fine mottles are there too and some of them clump together to form a coarse mottle of roughly $7000 \mathrm{~km}$ diameter. There is also enhanced emission at the boundary by magneto-hydrodynamic heating accentuated by the piling up of the magnetic field swept up by the horizontal flow within the domain of the supergranule. The three principal sources of calcium emission are thus the plage, the network boundaries and the fine mottle.

In a recent paper (Bappu and Sivaraman, 1971) we have carried through a study that shows which of these three features is responsible for the K-line width-absolute magnitude relation of Wilson and Bappu (1957). We carried out this study from a single frame of excellent quality which we had obtained in a $\mathrm{K}$-line time sequence of a specific region at the centre of the disc. A spectroheliogram taken soon after this exposure showed us the location of the slit in terms of the $K_{232}$ network and other features seen in the light of ionized calcium. The dispersion of the spectrogram was $9.4 \mathrm{~mm} \AA^{-1}$, almost a hundredfold larger than what Wilson and I had used earlier in the stellar case! It is well to realize however, that the $\mathrm{K}$ emission feature in the case of the Sun is seldom seen at dispersions lower than about $4 \AA \mathrm{mm}^{-1}$. At the large values of dispersion 
employed currently for the study of solar inhomogeneities, one sees a large amount of detail, specially when the spectrogram is obtained during the moments of good seeing. One sees numerous emission streaks of sizes in the range 1-2", mostly with emission greater in the violet portion of $\mathrm{K}_{2}$ than in the red or $I_{\mathrm{K} 2 \mathrm{~V}}>I_{\mathrm{K} 2 \mathrm{R}}$. About one out of every five such emission streaks have $I_{\mathrm{K} 2 \mathrm{~V}}<I_{\mathrm{K} 2 \mathrm{R}}$. About one in twenty have the two portions equal and it is very seldom indeed that both $\mathrm{K}_{2 \mathrm{~V}}$ and $\mathrm{K}_{2 \mathrm{R}}$ are absent simultaneously. Our study shows a typical characteristic of $\mathrm{K}_{2}$ emission from a quiet region of the Sun to be the case $I_{\mathrm{K} 2 \mathrm{~V}}>I_{\mathrm{K} 2 \mathrm{R}}$. We interpret the changes from this normal aspect as due to the action of two types of absorbing components that are present on the same scale of inhomogeneity in sizes as the bright features. One component with minor Doppler displacements acting on the normal $\mathrm{K}_{232}$ profile produces the changes observed between $I_{\mathrm{K} 2 \mathrm{~V}}$ and $I_{\mathrm{K} 2 \mathrm{R}}$ relatively enhancing one at the expense of the other. The other component arises from what we call 'dark condensations' with down flowing velocities of $5-8 \mathrm{~km} \mathrm{~s}^{-1}$ and sizes of about $5000 \mathrm{~km}$. These dark condensations can be seen easily on any good $\mathrm{K}_{3}$ spectroheliogram and in our opinion give rise to the situation $\mathrm{K}_{2 \mathrm{R}}=0$.

These details of intensity fluctuations are of interest to the stellar case where we necessarily measure the integrated characteristics of $\mathrm{K}_{2}$ emission and all those features that upset the intensity ratio between $I_{\mathrm{K} 2 \mathrm{~V}}$ and $I_{\mathrm{K} 2 \mathrm{R}}$. In cases like $\alpha$ Bootis it is well to remember this aspect as one possibility that we could utilize to explain changes in the relative intensities of the $\mathrm{K}_{2}$ emission components.

Sivaraman and $\mathrm{I}$ have also measured the widths along the dispersion of all the double peaked emission features that we have on this particular frame. We measured the separations of the emission peaks as well as the separation of the minima between the emission feature and the regular $\mathrm{K}$ absorption feature. We call the former a $\mathrm{K}_{2}$ width and the latter a $K_{1}$ width. When we plot a histogram of the frequency of the $K_{2}$ widths we find a remarkable uniqueness of the value of the $K_{2}$ width about a particular value of $26 \mathrm{~km} \mathrm{~s}^{-1}$. The $\mathrm{K}_{1}$ widths do not show such a sharp clustering about a single value; however, they also have a mean characteristic spacing giving rise to a $K_{1}$ witdh of $41 \mathrm{~km} \mathrm{~s}^{-1}$. Since the intensities in the solar case of $I_{\mathrm{K}_{2 \mathrm{~V}}}, I_{\mathrm{K}_{2 \mathrm{R}}}$ are close to $I_{\mathrm{K}_{1 \mathrm{~V}}}$ and $I_{\mathrm{K}_{1 \mathrm{R}}}$ we get a width at half intensity of $33.5 \mathrm{~km} \mathrm{~s}^{-1}$, by taking a straight mean of the values given above. Compare this with the value of Wilson and Bappu (1957) obtained from an 'averaged' disc spectrogram of $34 \mathrm{~km} \mathrm{~s}^{-1}$ and by a micrometer setting on the emission feature. Since the sun fits well on the Wilson-Bappu relation with a measured width of $34 \mathrm{~km} \mathrm{~s}^{-1}$ it is clear that bright points of $\mathrm{K} 2$ emission are the principal contributors to the line width-absolute magnitude relation. The intensity scans perpendicular to the dispersion show that they have typical sizes of the order of 1-2". Hence, we conclude that these bright points that enable the sun to follow the line width-absolute magnitude relation are the fine mottles seen on a good quality spectroheliogram. We stabilize this identification from a comparative study of the auto correlation function obtained from intensity scans perpendicular to the dispersion as well as that obtained on a two dimension scale from a high quality $\mathrm{K}_{232}$ spectroheliogram. Both these give full widths at half maximum of $7000 \mathrm{~km}$. We 
conclude that the average spacing between the bright streaks on the spectrogram are the same as that of the fine mottles on the two dimension spectroheliogram. The argument thus secures our identification.

The value of $7000 \mathrm{~km}$ is the spacing between the brightest features within the supergranular network and also that between the bright streaks on the spectrogram. The spacing between these streaks also shows the emission peaks but of lower intensity. We may ascribe these to an unresolved background of fine mottles that awaits detection with the improved resolution techniques of the future.

The life times of the emission streaks on the spectrogram and of the fine mottles on spectroheliograms provide an additional confirmation of identity. Both have values around $200 \mathrm{~s}$ and hence both are identical.

The behaviour of emission peak separations on plage regions have been known for a very long time. From the stand point of our terminology the $\mathrm{K}_{2}$ widths decrease with enhanced plage intensity and proximity to the seat of the centre of activity. Elske Smith (1960) has expressed this quantitatively. She also finds a minor change in the widths of the emission feature as one goes to plages with enhanced magnetic fields. In terms of the discussion above, it is seen that the plage regions offer little hope of being the source of a unique value for the $\mathrm{K}_{2}$ separations similar to the case of the fine mottles.

A similar situation prevails at the boundary of the supergranular network. Calcium emission here is greatly enhanced and has a noticeable contribution from the magnetic fields accumulated by virtue of the supergranular flow. The $\mathrm{K}_{2}$ width here is in the neighbourhood of $20 \mathrm{~km} \mathrm{~s}^{-1}$ instead of a value of $26 \mathrm{~km} \mathrm{~s}^{-1}$ for the fine mottles. It is clear that supergranular boundaries do not have any appreciable contribution to the unique value of the solar case in the relation of Wilson and Bappu (1957).

We are thus left with the bright fine mottle as the principal contributor to the K-line width-absolute magnitude relation. The fine mottle is not known to display any appreciable longitudinal field greater than the limit of photographic detectability. The bright mottle is thus, by some cause as yet unknown, a manifestation in the chromosphere of the mechanical energy dissipation from the convective layers below. It has characteristics prescribed by a combination of the fundamental parameters of the star.

In the calibration of the $\mathrm{K}$-line width-absolute magnitude relation, it is necessary to assign a value for the sun that is truly representative of it in integrated light. Wilson and Bappu had 'averaged' a sample of the disc that gave them the value of $34 \mathrm{~km} \mathrm{~s}^{-1}$ utilized in the calibration of the relation. Such a spectrum would yield a totality of bright mottle spectral features with the systemic velocities of the different mottles all added together to give an integrated profile. Sivaraman and $I$ have obtained several $\mathrm{K}$-line spectra at high dispersion in truly integrated light with the same spectrograph used for the high resolution study. The integrated spectrum thus obtained has an emission line width in excess of the corresponding value obtained by averaging the mottle spectra. Clearly we see here the contribution by solar rotation to the integrated characteristics. The emission line width in the solar case is closer to $36 \mathrm{~km} \mathrm{~s}^{-1}$ than the previously utilized value by Wilson and Bappu (1957). 
Calcium emission in the stars can be produced in several different ways. In binaries, we have often the case of emission from the gas flow within the system. Magnetic fields in star spots could produce enhanced calcium emission in star plages considerably more virulent than in the solar case. Also, from an extrapolation of the solar case we have the field of the bright mottle that is a feature of the convection below the stellar photosphere and which gives rise, according to our identification, to the Wilson-Bappu relation. The emission by magnetic field stimulated plages can be quite different from the emission of the quiet background, and cause an apparent deviation from the line width- $M_{v}$ relation. There can also be gaseous outflow which gives a deeper $\mathrm{K}_{3}$ and which indicates an abnormality in the emission origin. The case of Canopus seems to fall in line with such a possibility. K-line emission in the borderline cases of the earlier spectral types should be interpreted with caution, especially when they occur in the spectral domain where convection does not have a good role to play.

\section{References}

Bappu, M. K. V. and Sivaraman, K. R.: 1971, Solar Phys. 17, 316.

Smith, E. V. P.: 1960, Astrophys. J. 132, 202.

Wilson, O. C. and Bappu, M. K. V.: 1957, Astrophys. J. 125, 661.

\section{DISCUSSION}

Blaauw: To what extent might variations in the phenomena causing the $\mathrm{Ca}^{+}$reversals affect the widths and hence the derived luminosities?

Bappu: The presence of centres of activity and hence $\mathrm{Ca}^{+}$plages would undoubtedly enhance the overall intensity of the emission. Such enhancements independently or coupled with a dominant flow of absorbing gas outward or inward are likely to produce changes with time of the relative intensities of the emission components $\mathrm{K}_{2 \mathrm{~V}}$ and $\mathrm{K}_{2 \mathrm{R}}$. The contribution to the widths as measured presently for luminosity determinations are likely to be small; they fall within the present limits of uncertainty. The case of $\alpha$ Bootis supports such a conjecture since its $\mathrm{K}$-line width values do not seem to be affected by the changes seen in the $K_{2}$ emission. 